\title{
Effective reaction rates of a thin catalyst layer
}

\author{
Michael Lenzinger and Ben Schweizer
}

Preprint 2008-22

November 2008

Fakultät für Mathematik

Technische Universität Dortmund

Vogelpothsweg 87

44227 Dortmund

$\underline{\text { tu-dortmund.de/MathPreprints }}$ 



\title{
Effective reaction rates of a thin catalyst layer
}

\author{
Michael Lenzinger and Ben Schweizer ${ }^{1}$
}

October 31, 2008

\begin{abstract}
The catalyst layer in a fuel cell can be described with the help of a system of reaction diffusion equations for the protonic overpotential and the oxygen concentration. The Tafel equation gives an exponential law for the reaction rate, the Tafel slope is a coefficient in this law. We present a rigorous thin layer analysis for two reaction regimes. In the case of thin catalyst layers, the original Tafel slope enters an effective boundary condition. In the case of large protonic overpotentials we derive the effect of a double Tafel slope, essentially a consequence of a thin active region within the catalyst layer.
\end{abstract}

Keywords: thin layer analysis, reaction diffusion, effective boundary conditions, fuel cells, double Tafel slope

\section{Introduction}

The proton exchange membrane (PEM) fuel cell is an electrochemical device that converts chemical into electrical energy. The membrane separates the two gases hydrogen and oxygen. Protons diffuse through the membrane and react in the cathode catalytic layer with oxygen. The electrons travel through the external circuit and thus provide the electric current. The membrane is separated from the flow chanels by gas diffusion layers (GDLs) which are chemically inactive porous media.

The processes in the catalytic layer can be described with a system of two reaction diffusion equations, see e.g. $[2,5,9,10,16]$. We follow the notation of $[2]$ and denote the protonic overpotential by $\eta$ and the oxygen concentration by $C$. The stationary situation is described by the following system for $\eta$ and $C$.

$$
\begin{aligned}
\nabla \cdot\left(M_{\eta} \nabla \eta\right) & =\mu_{\eta} C e^{\eta / b} \\
\nabla \cdot\left(M_{C} \nabla C\right) & =\mu_{C} C e^{\eta / b} .
\end{aligned}
$$

\footnotetext{
${ }^{1}$ Fakultät für Mathematik, TU Dortmund, Vogelpothsweg 87, 44227 Dortmund, Germany. michael.lenzinger@tu-dortmund.de and ben.schweizer@tu-dortmund.de
} 
In these equations $M_{\eta}$ and $M_{C}$ are effective diffusion constants, $\mu_{\eta}$ and $\mu_{C}$ are effective reaction constants. The equations include a nonlinear source term of exponential form. This source expresses the Tafel law: the reaction is proportional to $C e^{\eta / b}$, where the positive constant $b$ is the Tafel slope. Equations (1.1) and (1.2) are effective equations that can be derived from microscopic models, see e.g. [2, 5, 9] for physical derivations and [11] for a rigorous analysis with homogenization techniques. Definitions of non-dimensional coefficients and typical values are available in $[2,11]$.

The interesting effect of a doubling of the Tafel slope for large overpotentials can be observed experimentally and in simulations $[7,9,14,15]$. The most accessible averaged quantity is that of the total production $F$, see the definition below. The observation is that, in a wide range of parameter values, $F$ obeys the exponential law (I) for some Tafel slope $b$, where $k$ is a reference value of the potential $\eta$. Instead, for extreme values of $k$, one observes the dependence (II). This effect is known as the doubling of the Tafel slope. We mention that even a quadruple Tafel slope is reported in [9].

$$
\text { (I) } F(k) \sim e^{k / b} \quad \text { (II) } F(k) \sim e^{k /(2 b)}
$$

In this contribution we derive laws (I) and (II) in different regimes of the fuel cell parameters. We always start from the reaction diffusion system (1.1), (1.2). In the limit of a thin catalyst layer we derive (I), in the limit of large potential we derive (II). The precise statements are given in Theorem 1 below.

The limit analysis leading to (I) follows the lines of many classical applications of asymptotic analysis. In [6], a large source term in a small layer results in an effective boundary condition for the bulk equation. A similar effect appears in $[1,13]$ in an homogenization limit. In the much more involved equations of elasticity, one is interested in equations on the lower dimensional limit of the thin layers, see e.g. [12]. Reduced dynamics in thin films appear in micromagnetics, see e.g. [4]. Another application are phase field models and their limits, see e.g. [3, 8]. We mentioned prototypical articles and refer also to the references therein.

Concerning (II) we are not aware of any rigorous results. The effect of a double Tafel slope is specific to the exponential form of the nonlinearity.

\section{Main results}

We describe here our system of equations in the one-dimensional case. The diffusion constants $M_{\eta}$ and $M_{C}$ can be incorporated into new effective reaction rates $\mu_{\eta}$ and $\mu_{C}$. A boundary value $C_{1} \geq 0$ for the oxygen concentration at the oxygen flow chanel is assumed to be given. We introduce two numbers that 
determine the overall regime of the reaction processes: The (small) number $\delta>0$ denotes the thickness of the catalyst layer, the number $k$ a boundary value for the protonic potential (on the side of the PEM). We consider the overpotential $\eta=\eta^{\delta, k}$ and the oxygen concentration $C=C^{\delta, k}$. The thin catalyst layer occupies the domain $\Omega_{\delta}=(0, \delta)$, the total flow domain is $\Omega_{0}=(0,1)$ and consists of the catalyst layer and the adjacent GDL. We consider the equation for $\eta$ in $\Omega_{\delta}$,

$$
\begin{aligned}
\partial_{x}^{2} \eta & =\mu_{\eta} C e^{\eta / b} \quad \text { in } \Omega_{\delta}, \\
\eta(0) & =k, \quad \partial_{x} \eta(\delta)=0,
\end{aligned}
$$

coupled to the equation for $C$ in $\Omega_{0}$,

$$
\begin{aligned}
\partial_{x}^{2} C & =\mu_{C} C e^{\eta / b} \mathbf{1}_{\Omega_{\delta}} \quad \text { in } \Omega_{0}, \\
\partial_{x} C(0) & =0, \quad C(1)=C_{1},
\end{aligned}
$$

where $\mathbf{1}_{\Omega_{\delta}}$ denotes the characteristic function of $\Omega_{\delta}$. Of particular interest is the total production or protonic flux

$$
F_{\delta, k}:=\int_{0}^{\delta} \mu_{\eta} C e^{\eta / b}=-\partial_{x} \eta(0) .
$$

For the one-dimensional case our results are collected in the following theorem. It provides two effective models, one for thin catalyst layers, another for high potentials. In the first regime we must scale the reaction constants with $\delta$ in order to have a finite production in the thin layer; in the corresponding effective model the catalytic layer is replaced by a boundary condition that includes the Tafel law. In the second regime we derive an explicit formula for the total production in the thin layer. The formula shows the doubling of the Tafel slope.

Theorem 1. Let $C^{\delta, k}$ and $\eta^{\delta, k}$ be solutions to equations (1.3)-(1.6) for $C_{1} \geq 0$. Then, for a universal constant $q_{1}>0$, the following limits hold.

(i) For $\mu_{\eta}=\mu_{\eta}^{*} / \delta, \mu_{C}=\mu_{C}^{*} / \delta$, fixed $k, \mu_{\eta}^{*}, \mu_{C}^{*}>0$, and any sequence $\delta \rightarrow 0$ there holds $C^{\delta, k} \rightarrow C^{0, k}$ weakly in $H^{1}\left(\Omega_{0}\right)$, where $C^{0, k}$ is determined by the effective equation

$$
\begin{aligned}
\partial_{x}^{2} C^{0, k} & =0 \quad \text { in } \Omega_{0}, \\
\partial_{x} C^{0, k}(0) & =\mu_{C}^{*} C^{0, k}(0) e^{k / b}, \quad C^{0, k}(1)=C_{1} .
\end{aligned}
$$

The production rate satisfies

$$
\frac{F_{\delta, k}}{e^{k / b}} \rightarrow \mu_{\eta}^{*} C^{0, k}(0)=\frac{\mu_{\eta}^{*} C_{1}}{1+\mu_{C}^{*} e^{k / b}} \quad \text { as } \delta \rightarrow 0 .
$$


(ii) Given a sequence $k \rightarrow \infty$ and parameters $\mu_{\eta}>0, \delta=\delta(k), \mu_{C}=\mu_{C}(k)$ with $\delta(k) \rightarrow \delta_{0} \in[0,1), \delta(k) e^{k /(2 b)} \rightarrow \infty, \mu_{C}(k) e^{k /(2 b)} \rightarrow \bar{\mu}_{C} \geq 0$, and $\delta(k)^{5} e^{5 k /(2 b)} \mu_{C}(k) \rightarrow 0$. Then the total production satisfies

$$
\frac{F_{\delta, k}}{e^{k /(2 b)}} \rightarrow q_{1} \sqrt{b \mu_{\eta} \bar{C}} \text { for } k \rightarrow \infty
$$

where $\bar{C}$ is defined by

$$
\bar{C}+q_{1} \bar{\mu}_{C}\left(1-\delta_{0}\right) \sqrt{b \bar{C} / \mu_{\eta}}=C_{1} .
$$

We remark that solutions $C^{0, k}$ of (1.7)-(1.8) are affine, which immediately implies the equality in (1.9). To illustrate the two cases of the theorem, typical solutions are sketched in Figure 1. For an even more direct comparison of the results we note the following. In case (i), for $\mu_{C}^{*} \rightarrow 0$, formula (1.9) simplifies to (1.11), whereas in case (ii), for $\bar{\mu}_{C} \rightarrow 0$, formula (1.10) simplifies to (1.12),

$$
\begin{aligned}
\frac{F_{\delta, k}}{e^{k / b}} & \rightarrow \mu_{\eta}^{*} C_{1} \text { as } \delta \rightarrow 0, \\
\frac{F_{\delta, k}}{e^{k /(2 b)}} & \rightarrow q_{1} \sqrt{b \mu_{\eta} C_{1}} \text { for } k \rightarrow \infty .
\end{aligned}
$$

In Section 2 we analyze system (1.3)-(1.6) in the $N$-dimensional setting. The effective equations are derived, the one-dimensional case provides (i) of the theorem. The proof of (ii) is the aim of Section 3. We note that this second result concerns small reaction constants $\mu_{C}$. Numerical calculations for the universal constant provide $q_{1} \approx 1.413$.

\section{Thin catalyst layers and moderate potential}

In this section we prove the first part of Theorem 1 on rectangles in $\mathbb{R}^{N}$. The space dimension $N$ is arbitrary, we present results and proofs in the physical case $N=3$. For the boundary condition of $\eta$ corresponding to (1.4) we write $\eta_{0}$ instead of $k$. In this section, the function $\eta_{0}$ is kept fixed and we suppress the superscript $k$. Accordingly, we write $C^{\delta}, C^{0}$ and $F_{\delta}$ instead of $C^{\delta, k}, C^{0, k}$ and $F_{\delta, k}$.

We analyze the case that the catalyst layer is thin and the reaction rates are large. The two quantities are balanced in such a way that the total production remains finite, i.e. $\mu_{\eta}$ and $\mu_{C}$ are of order $1 / \delta$, we write $\mu_{\eta}=\mu_{\eta}^{*} / \delta$ and $\mu_{C}=$ $\mu_{C}^{*} / \delta$. This assumption refers to fuel cells operating in a regime of high current density. Referring to [2] we have, in SI units, $\delta$ of order $10^{-6}$, and $\mu_{\eta}$ and $\mu_{C}$ of 

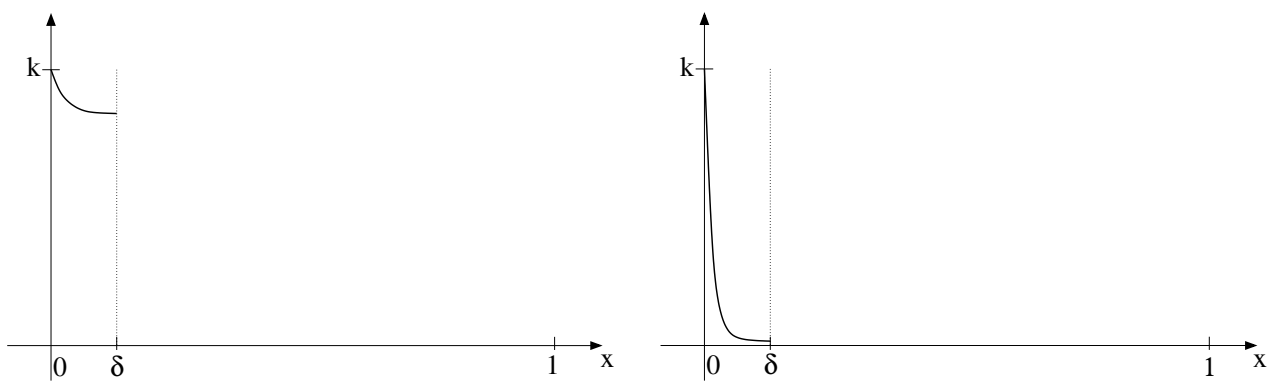

Figure 1: Sketch of solutions $\eta^{\delta, k}$ of the one-dimensional system (1.3)-(1.6). On the left we sketch the situation of (i), treated in Section 2: the solution for small $\delta$ and moderate $k$ is almost constant in the $\delta$-layer. On the right we sketch case (ii), treated in Section 3: the fast decay of the solution reduces the width of the active layer and results in the double Tafel slope.

the order $10^{4}$ (we used a current density $i_{0}$ of order $10^{-2}$, and refer once more to the collection of typical values in [11]).

We investigate the geometry sketched in Figure 2. The domain of interest is composed of the homogenized catalyst layer $\Omega_{\delta}=\left(0, L_{1}\right) \times\left(0, L_{2}\right) \times(0, \delta)$ of thickness $\delta$, and the gas diffusion layer $\Omega_{0 \delta}=\left(0, L_{1}\right) \times\left(0, L_{2}\right) \times\left(\delta, L_{3}\right)$. The reactions are confined to the layer $\Omega_{\delta}$, in the limit $\delta \rightarrow 0$ we expect an effective equation posed on the entire domain $\Omega_{0}=\left(0, L_{1}\right) \times\left(0, L_{2}\right) \times\left(0, L_{3}\right)$ with a nonhomogeneous boundary condition on the boundary $\Sigma_{0}=\left(0, L_{1}\right) \times\left(0, L_{2}\right) \times\{0\}$ at the reactive side. As additional notation we use $\Sigma_{\delta}=\left(0, L_{1}\right) \times\left(0, L_{2}\right) \times\{\delta\}$ for the interface between gas diffusion layer and catalyst layer, and $\Sigma_{1}=\left(0, L_{1}\right) \times$ $\left(0, L_{2}\right) \times\left\{L_{3}\right\}$ for the non-reactive boundary, and denote by $n$ the normal vector to $\Omega_{0 \delta}$. For simplicity of notation we always impose periodicity conditions on the remaining boundaries. We study the following macroscopic equations for the overpotential $\eta$ and the oxygen concentration $C$. To emphasize the dependence on $\delta$ we write $\eta^{\delta}: \Omega_{\delta} \rightarrow \mathbb{R}$ for the overpotential and $C^{\delta}: \Omega_{0} \rightarrow \mathbb{R}$ for the oxygen concentration.

$$
\begin{aligned}
\nabla \cdot\left(M_{\eta} \nabla \eta^{\delta}\right) & =\frac{1}{\delta} \mu_{\eta}^{*} C^{\delta} e^{\eta^{\delta} / b} & & \text { in } \Omega_{\delta} \\
\eta^{\delta} & =\eta_{0} & & \text { on } \Sigma_{0} \\
n \cdot\left(M_{\eta} \nabla \eta^{\delta}\right) & =0 & & \text { on } \Sigma_{\delta} \\
\nabla \cdot\left(M_{C} \nabla C^{\delta}\right) & =\frac{1}{\delta} \mu_{C}^{*} C^{\delta} e^{\eta^{\delta} / b} \mathbf{1}_{\Omega_{\delta}} & & \text { in } \Omega_{0} \\
n \cdot\left(M_{C} \nabla C^{\delta}\right) & =0 & & \text { on } \Sigma_{0} \\
C^{\delta} & =C_{1} & & \text { on } \Sigma_{1}
\end{aligned}
$$



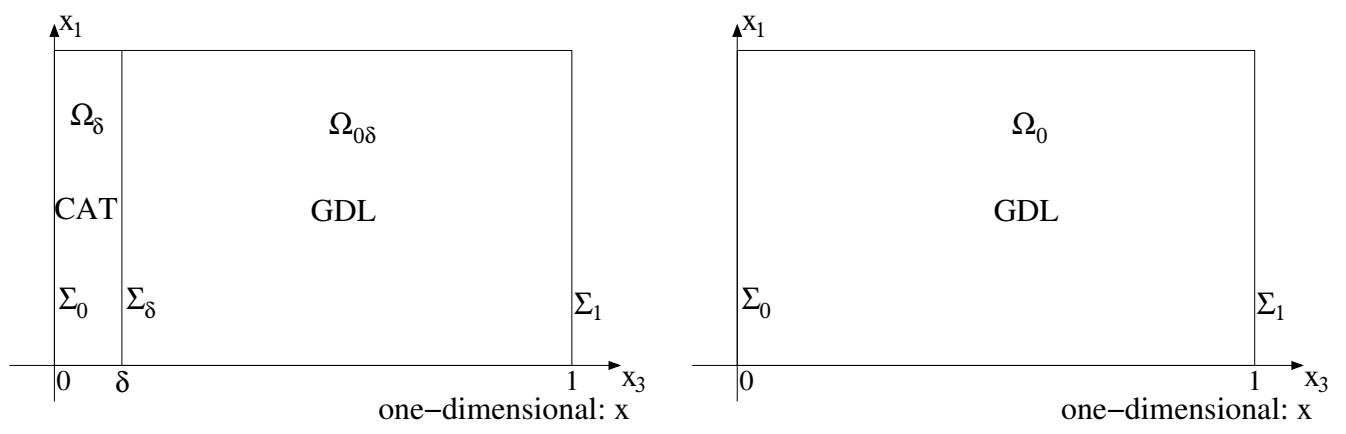

Figure 2: Geometry of the $\delta$-problem and the effective domain for $L_{3}=1$. In the limit domain $\Omega_{0}=\Omega_{0 \delta} \cup \Sigma_{\delta} \cup \Omega_{\delta}$, the reaction term appears as a nonlinear boundary condition on the left boundary $\Sigma_{0}$.

For the derivation of effective equations it is useful to introduce the averaged overpotential as

$$
\bar{\eta}^{\delta}\left(x_{1}, x_{2}\right)=f_{0}^{\delta} \eta^{\delta}\left(x_{1}, x_{2}, y\right) d y, \quad \bar{\eta}^{\delta}: \Sigma_{0} \rightarrow \mathbb{R}
$$

We will identify functions $\varphi: \Sigma_{\delta} \rightarrow \mathbb{R}$ with $\varphi: \Sigma_{0} \rightarrow \mathbb{R}$.

Theorem 2. (Effective equations in thin catalyst layers) Let $\eta^{\delta}, C^{\delta}$ be a weak solution of (2.1)-(2.6) and let the boundary conditions be given by $\eta_{0} \in H^{1}\left(\Sigma_{0}\right) \cap$ $L^{\infty}\left(\Sigma_{0}\right)$ and $C_{1} \in H^{1}\left(\Omega_{0}\right) \cap L^{\infty}\left(\Omega_{0}\right), C_{1} \geq 0$. Then, for every sequence $\delta \rightarrow 0$ there holds

$$
\begin{gathered}
\bar{\eta}^{\delta} \rightarrow \eta_{0} \quad \text { strongly in } L^{2}\left(\Sigma_{0}\right) \\
C^{\delta} \rightarrow C^{0} \quad \text { weakly in } H^{1}\left(\Omega_{0}\right)
\end{gathered}
$$

where $C^{0}$ satisfies weakly the effective equations

$$
\begin{aligned}
& \nabla \cdot\left(M_{C} \nabla C^{0}\right)=0 \quad \text { in } \Omega_{0} \\
& n \cdot\left(M_{C} \nabla C^{0}\right)=-\mu_{C}^{*} C^{0} e^{\eta_{0} / b} \quad \text { on } \Sigma_{0}
\end{aligned}
$$

with the other boundary conditions remaining unchanged. The production rate satisfies

$$
F_{\delta}=\int_{0}^{\delta} \mu_{\eta} C^{\delta} e^{\eta^{\delta} / b} \rightarrow \mu_{\eta}^{*} \int_{\Sigma_{0}} C^{0} e^{\eta_{0} / b} \quad \text { for } \delta \rightarrow 0
$$


Proof. We start with the a priori estimates for the sequence $\left(C^{\delta}, \eta^{\delta}\right)$ of solutions.

Uniform bounds. The maximum principle can be applied to the oxygen concentration $C^{\delta}$ since the factor $\frac{\mu_{C}^{*}}{\delta} e^{\eta^{\delta} / b}$ on the right-hand side of (2.4) is nonnegative. In particular, we obtain

$$
0 \leq C^{\delta} \leq\left\|C_{1}\right\|_{L^{\infty}\left(\Omega_{0}\right)} .
$$

The non-negativity of $C^{\delta}$ implies that the right-hand side of (2.1) is nonnegative. The maximum principle for $\eta^{\delta}$ implies the upper bound

$$
\sup _{\Omega_{\delta}} \eta^{\delta} \leq \sup _{\Sigma_{0}} \eta_{0} .
$$

Note that we only have an upper bound for $\eta^{\delta}$, but this already provides a uniform bound of the exponential term $e^{\eta^{\delta} / b}$.

Energy estimates. We extend the boundary values $\eta_{0}$ trivially to the function $\eta_{0}\left(x_{1}, x_{2}, x_{3}\right)=\eta_{0}\left(x_{1}, x_{2}\right)$ and multiply (2.1) with $\eta^{\delta}-\eta_{0}$. An integration over $\Omega_{\delta}$ yields

$$
\int_{\Omega_{\delta}} M_{\eta} \nabla \eta^{\delta} \cdot \nabla \eta^{\delta}+\frac{1}{\delta} \int_{\Omega_{\delta}} \mu_{\eta}^{*} C^{\delta} e^{\eta^{\delta} / b} \eta^{\delta}=\int_{\Omega_{\delta}} M_{\eta} \nabla \eta^{\delta} \cdot \nabla \eta_{0}+\frac{1}{\delta} \int_{\Omega_{\delta}} \mu_{\eta}^{*} C^{\delta} e^{\eta^{\delta} / b} \eta_{0} .
$$

The uniform bounds for $C^{\delta}$ and $e^{\eta^{\delta} / b}$ provide the boundedness of the second integral on the right-hand side. Since the function $\xi e^{\xi}$ is bounded from below for all $\xi \in \mathbb{R}$ we additionally have a uniform lower bound for the second integral on the left-hand side. We conclude that the $H^{1}\left(\Omega_{\delta}\right)$-norm of $\eta^{\delta}$ is bounded, in particular $\left\|\nabla \eta^{\delta}\right\|_{L^{2}\left(\Omega_{\delta}\right)} \leq c$. Furthermore, we have by Poincaré's inequality

$$
\begin{aligned}
\left\|\bar{\eta}^{\delta}-\eta_{0}\right\|_{L^{2}\left(\Sigma_{0}\right)} & =\left\|f_{0}^{\delta}\left(\eta^{\delta}-\eta_{0}\right)\right\|_{L^{2}\left(\Sigma_{0}\right)} \leq \frac{1}{\delta^{1 / 2}}\left\|\eta^{\delta}-\eta_{0}\right\|_{L^{2}\left(\Omega_{\delta}\right)} \\
& \leq \delta^{1 / 2}\left\|\nabla\left(\eta^{\delta}-\eta_{0}\right)\right\|_{L^{2}\left(\Omega_{\delta}\right)} \leq c \delta^{1 / 2},
\end{aligned}
$$

and therefore the strong convergence of $\bar{\eta}^{\delta}$, i.e. (2.7).

We now multiply (2.4) with $C^{\delta}-C_{1}$ and integrate over $\Omega_{0}$. We obtain

$$
\int_{\Omega_{0}} M_{C} \nabla C^{\delta} \cdot \nabla C^{\delta}+\int_{\Omega_{\delta}} \frac{1}{\delta} \mu_{C}^{*} C^{\delta} e^{\eta^{\delta} / b}\left(C^{\delta}-C_{1}\right)=\int_{\Omega_{0}} M_{C} \nabla C^{\delta} \cdot \nabla C_{1} .
$$

The second integral on the left hand side is bounded from below by the uniform bounds for $C^{\delta}, C_{1}$, and $e^{\eta^{\delta} / b}$. Hölder's estimate yields the boundedness of $C^{\delta}$ in $H^{1}\left(\Omega_{0}\right)$. In particular, we can select a weakly convergent subsequence with a limit as in (2.8). 
The equations for $C^{0}$. Multiplication of (2.4) with $\varphi \in \mathcal{D}\left(\Omega_{0} \cup \Sigma_{0}\right)$ and an integration over $\Omega_{0}$ yields

$$
\int_{\Omega_{0}} M_{C} \nabla C^{\delta} \cdot \nabla \varphi+\int_{\Omega_{\delta}} \frac{1}{\delta} \mu_{C}^{*} C^{\delta} e^{\eta^{\delta} / b} \varphi=0
$$

In the first integral we can pass to the limit by the weak convergence of $C^{\delta}$. Lemma 1 below provides the nontrivial limit

$$
\int_{\Omega_{\delta}} \frac{1}{\delta} \mu_{C}^{*} C^{\delta} e^{\eta^{\delta} / b} \varphi \rightarrow \int_{\Sigma_{0}} \mu_{C}^{*} C^{0} e^{\eta_{0} / b} \varphi
$$

for $\delta \rightarrow 0$. Thus, the limit equation of $(2.13)$ is

$$
\int_{\Omega_{0}} M_{C} \nabla C^{0} \cdot \nabla \varphi+\int_{\Sigma_{0}} \mu_{C}^{*} C^{0} e^{\eta_{0} / b} \varphi=0
$$

which is the weak form of (2.9)-(2.10). The limit problem has a unique solution, therefore the whole sequence converges.

The limit expression for the production rate. Setting $\varphi=1$ in (2.14) yields

$$
F_{\delta}=\int_{0}^{\delta} \mu_{\eta} C^{\delta} e^{\eta^{\delta} / b}=\mu_{\eta}^{*} \int_{0}^{\delta} C^{\delta} e^{\eta^{\delta} / b} \rightarrow \mu_{\eta}^{*} \int_{\Sigma_{0}} C^{0} e^{\eta_{0} / b} \quad \text { for } \delta \rightarrow 0
$$

This was the claim.

In the above proof we used a convergence result which is the consequence of the fact that $\eta^{\delta}$ has small variations in the thin layer $\Omega_{\delta}$.

Lemma 1. Let $\eta^{\delta}, C^{\delta}$ be the solutions of (2.1)-(2.6) as in Theorem 2, with boundary condition $\eta_{0}$ and limit $C^{0}$, and let $\varphi \in C^{0}\left(\bar{\Omega}_{0}\right)$. Then

$$
f_{\Omega_{\delta}} C^{\delta} e^{\eta^{\delta} / b} \varphi \rightarrow \int_{\Sigma_{0}} C^{0} e^{\eta_{0} / b} \varphi \quad \text { for } \delta \rightarrow 0
$$

Proof. First step. We claim that the averages of $C^{\delta}$ converge weakly,

$$
\left.f_{0}^{\delta} C^{\delta}(., y) d y \rightarrow C^{0}\right|_{\Sigma_{0}} \text { weakly in } L^{2}\left(\Sigma_{0}\right) \text { for } \delta \rightarrow 0
$$

Indeed, the trace theorem provides the boundedness of every restriction of $C^{\delta}$ to a two-dimensional subset $\Sigma_{y}:=\left\{(x, y):(x, 0) \in \Sigma_{0}\right\}$ with the bound

$$
\left\|\operatorname{trace}_{\Sigma_{y}} C^{\delta}\right\|_{L^{2}} \leq c\left\|C^{\delta}\right\|_{H^{1}\left(\Omega_{0}\right)} \leq c
$$


Jensen's inequality implies that also the average of traces is bounded in $L^{2}$,

$$
\begin{aligned}
\left\|f_{0}^{\delta} C^{\delta}(., y) d y\right\|_{L^{2}\left(\Sigma_{0}\right)} & =\left\|\frac{1}{\delta} \int_{0}^{\delta} \operatorname{trace}_{\Sigma_{y}} C^{\delta}\right\|_{L^{2}\left(\Sigma_{0}\right)} \\
& \leq \frac{1}{\delta} \int_{0}^{\delta}\left\|\operatorname{trace}_{\Sigma_{y}} C^{\delta}\right\|_{L^{2}\left(\Sigma_{0}\right)} \leq c .
\end{aligned}
$$

The boundedness of $f_{0}^{\delta} C^{\delta}$ in $L^{2}\left(\Sigma_{0}\right)$ allows to choose a subsequence and a function $g \in L^{2}\left(\Sigma_{0}\right)$ with

$$
f_{0}^{\delta} C^{\delta} \rightarrow g \text { weakly in } L^{2}\left(\Sigma_{0}\right) \text { for } \delta \rightarrow 0 .
$$

It remains to identify $g=\operatorname{trace}_{\Sigma_{0}} C^{0}$, which also provides the convergence of the original sequence. To this end we let $\psi \in C_{0}^{1}\left(\Omega_{0} \cup \Sigma_{0}\right)$ be an arbitrary function and calculate

$$
\begin{aligned}
& \int_{\Sigma_{0}}\left(f_{0}^{\delta} C^{\delta}\right) \psi=\frac{1}{\delta} \int_{0}^{\delta} \int_{\Sigma_{y}} C^{\delta} \psi+O(\delta) \\
& =-\frac{1}{\delta} \int_{0}^{\delta} \int_{\left(0, L_{1}\right) \times\left(0, L_{2}\right) \times\left(y, L_{3}\right)} \frac{\partial}{\partial y}\left(C^{\delta} \psi\right)+O(\delta) \\
& \rightarrow-\int_{\Omega_{0}} \frac{\partial}{\partial y}\left(C^{0} \psi\right)=\int_{\Sigma_{0}} C^{0} \psi
\end{aligned}
$$

for $\delta \rightarrow 0$. The left hand side converges to $\int_{\Sigma_{0}} g \psi(., 0)$, we have therefore identified $g$ and (2.15) is shown.

Second step. For the auxiliary function

$$
u_{\delta}\left(x_{1}, x_{2}, y\right)=e^{\eta^{\delta}\left(x_{1}, x_{2}, y\right) / b}-e^{\eta_{0}\left(x_{1}, x_{2}\right) / b}
$$

we have due to Poincaré's inequality

$$
\frac{1}{\delta} \int_{\Omega_{\delta}}\left|u_{\delta}\right|^{2} \leq c \delta \int_{\Omega_{\delta}}\left|\nabla u_{\delta}\right|^{2} \rightarrow 0 .
$$

Here we used that in $\nabla e^{\eta^{\delta} / b}=\frac{1}{b} e^{\eta^{\delta} / b} \nabla \eta^{\delta}$ the exponent $\eta^{\delta}$ is uniformly bounded from above by the maximum principle and $\nabla \eta^{\delta}$ is uniformly bounded in $L^{2}\left(\Omega_{\delta}\right)$. We can now perform the calculation for the product. For $\left(x_{1}, x_{2}\right)$ fixed we find

$$
\begin{aligned}
\left(f_{0}^{\delta} C^{\delta} e^{\eta^{\delta} / b}\right) & =f_{0}^{\delta} C^{\delta} e^{\eta_{0} / b}+f_{0}^{\delta} C^{\delta}\left[e^{\eta^{\delta} / b}-e^{\eta_{0} / b}\right] \\
& =e^{\eta_{0} / b} f_{0}^{\delta} C^{\delta}+f_{0}^{\delta} C^{\delta} u_{\delta},
\end{aligned}
$$


and therefore, by (2.16),

$$
\begin{aligned}
f_{\Omega_{\delta}} C^{\delta} e^{\eta^{\delta} / b} \varphi & =\int_{\Sigma_{0}}\left(f_{0}^{\delta} C^{\delta} e^{\eta^{\delta} / b}\right) \varphi(\cdot, 0)+o(1) \\
& =\int_{\Sigma_{0}}\left\{\left(f_{0}^{\delta} C^{\delta}\right)\left(x_{1}, x_{2}\right) e^{\eta_{0}\left(x_{1}, x_{2}\right) / b} \varphi\left(x_{1}, x_{2}, 0\right)\right\} d x_{1} d x_{2}+o(1)
\end{aligned}
$$

for all $\varphi \in C^{0}\left(\bar{\Omega}_{0}\right)$. The weak $L^{2}\left(\Sigma_{0}\right)$-convergence of the $C^{\delta}$-average in $(2.15)$ provides the result.

\section{Thin catalyst layers at high potentials}

In this section we are interested in the effect of a double Tafel slope, case (ii) of Theorem 1. We have to analyze the one-dimensional system (1.3)-(1.6) in the limit of high potentials, $k \rightarrow \infty$. Omitting the dependence on $\delta$, we now write $\eta^{k}$ and $C^{k}$ for solutions. We recall that we are given a sequence $k \rightarrow \infty$ and that the parameters $\delta=\delta(k)$ and $\mu_{C}=\mu_{C}(k)$ satisfy $\delta(k)^{4} \mu_{C}(k) e^{5 k /(2 b)} \rightarrow 0$ and $\delta(k) e^{k /(2 b)} \rightarrow \infty$. Our aim is to derive (1.10).

Physically, the above asymptotic behavior of the parameters reflects the case that the oxygen diffusion is large enough to provide an almost constant oxygen concentration in the whole catalyst layer. This situation of large oxygen diffusion $D \sim 1 / \mu_{C}$ is also analyzed in [9].

In Subsection 3.1 we consider the case of a constant oxygen concentration $\bar{C} \in[0, \infty)$ in the whole catalyst layer, the corresponding potential is denoted by $\bar{\eta}^{k}$. We derive the limit of the production rate (1.10) in this simplified case.

In Subsection 3.2 we compare the solution for large oxygen diffusion with the solution of constant oxygen distribution. In the case of a large diffusion constant, the two solutions $\left(C^{k}, \eta^{k}\right)$ and $\left(\bar{C}, \bar{\eta}^{k}\right)$ are similar and we conclude the theorem.

\subsection{Production rate for constant oxygen concentration}

We assume here a constant oxygen concentration $\bar{C} \geq 0$ in the whole catalyst layer $(0, \delta)$. Equations (1.3)-(1.6) then reduce to the following ordinary differential equation for $\bar{\eta}^{k}$.

$$
\begin{aligned}
\partial_{x}^{2} \bar{\eta}^{k} & =\mu_{\eta} \bar{C} e^{\bar{\eta}^{k} / b} \quad \text { in }(0, \delta) \\
\bar{\eta}^{k}(0) & =k \\
\partial_{x} \bar{\eta}^{k}(\delta) & =0 .
\end{aligned}
$$


We are interested in the limit $k \rightarrow \infty$ and in a limit expression for the production rate

$$
\bar{F}_{k}=-\partial_{x} \bar{\eta}^{k}(0)=\mu_{\eta} \int_{0}^{\delta} \bar{C} e^{\bar{\eta}^{k} / b}
$$

We will analyze equation (3.1) with methods of ordinary differential equation theory. The expression (3.8) of Proposition 1 provides the double Tafel slope in the case of constant $C$.

Our result is based on the following transformation of (3.1). We write shorter $\eta=\bar{\eta}^{k}$ for the solution of (3.1) - (3.3) and introduce

$$
\theta=\theta_{k}:=\frac{e^{\eta / b}}{e^{k / b}} \quad \text { and } \quad y:=x e^{k /(2 b)} .
$$

In the $y$-coordinate, the right end-point of the equation is in $y_{0}=\delta e^{k /(2 b)}$. With $c=\mu_{\eta} \bar{C} / b$ we calculate

$$
\begin{aligned}
\partial_{x}^{2} \eta & =\partial_{x}^{2}\left[b \log \left(\theta e^{k / b}\right)\right]=\partial_{x}\left[b \frac{\partial_{x} \theta}{\theta}\right]=b e^{k / b} \partial_{y}\left[\frac{\partial_{y} \theta}{\theta}\right]=b e^{k / b}\left[\frac{\partial_{y}^{2} \theta}{\theta}-\frac{\left(\partial_{y} \theta\right)^{2}}{\theta^{2}}\right] \\
\mu_{\eta} \bar{C} e^{\eta / b} & =b e^{k / b} c \theta .
\end{aligned}
$$

We see that the $\eta$-equation (3.1) is equivalent to the $\theta$-equation (3.5). In the next proposition the ordinary differential equation (3.5) is analyzed. Note that initial conditions instead of boundary conditions are imposed for this result.

Proposition 1. Consider, for given $q, c \in[0, \infty)$, the ordinary differential equation

$$
\begin{aligned}
\partial_{y}^{2} \theta-\frac{1}{\theta}\left(\partial_{y} \theta\right)^{2} & =c \theta^{2} \\
\theta(0) & =1 \\
\partial_{y} \theta(0) & =-q
\end{aligned}
$$

for $\theta=\theta(y)$ on $y \in(0, \infty)$. Then there exists a critical value $q^{*}=q^{*}(c) \in(0, \infty)$ such that the solutions $\theta=\theta_{q}$ of (3.5)-(3.7) satisfy

For $q>q^{*}$, the solution $\theta=\theta_{q}$ is monotonically decreasing, for $q<q^{*}$, the solution $\theta=\theta_{q}$ is positive and unbounded.

The dependence of the critical value on $c$ is given by $q^{*}(c)=q_{1} \sqrt{c}$ with $q_{1}:=$ $q^{*}(1)$. Numerically, we determined $q_{1} \approx 1.413$.

The production rate of (3.4) satisfies

$$
\frac{\bar{F}_{k}}{e^{k /(2 b)}} \rightarrow b q^{*}(c)=q_{1} \sqrt{b \mu_{\eta} \bar{C}} \text { for } k \rightarrow \infty .
$$


Proof. First step. Properties of solutions. Let $\theta$ be a solution of (3.5) on a maximal interval of existence $\left(0, y_{q}\right)$. We note that $\theta$ can never reach 0 ; this can be concluded e.g. from the transformed equation (3.1) which, by the positivity of the right hand side, admits only linear growth to $-\infty$ for $\eta=b \log \left(\theta e^{k / b}\right)$. We furthermore note that $\theta$ is smooth on $\left(0, y_{q}\right)$.

The derivative $\partial_{y} \theta(0)=-q$ is non-positive, hence there exists a maximal $y_{\min } \in\left(0, y_{q}\right]$ such that $\theta$ is monotonically decreasing on $\left(0, y_{\min }\right)$. Assume that $y_{\text {min }}<y_{q}$ such that $\partial_{y} \theta\left(y_{\min }\right)=0$. In this case, the solution is monotonically increasing on $\left(y_{\min }, y_{q}\right)$; due to the quadratic nonlinearity, the solution is unbounded. We conclude that solutions have only two possibilities. (a) they are monotonically decreasing on $(0, \infty)$ or $(b)$ they are positive and decreasing on $\left(0, y_{\min }\right)$, and increasing and unbounded on $\left(y_{\min }, y_{q}\right)$. We refer to Figure 3 for a sketch of the phase portrait.

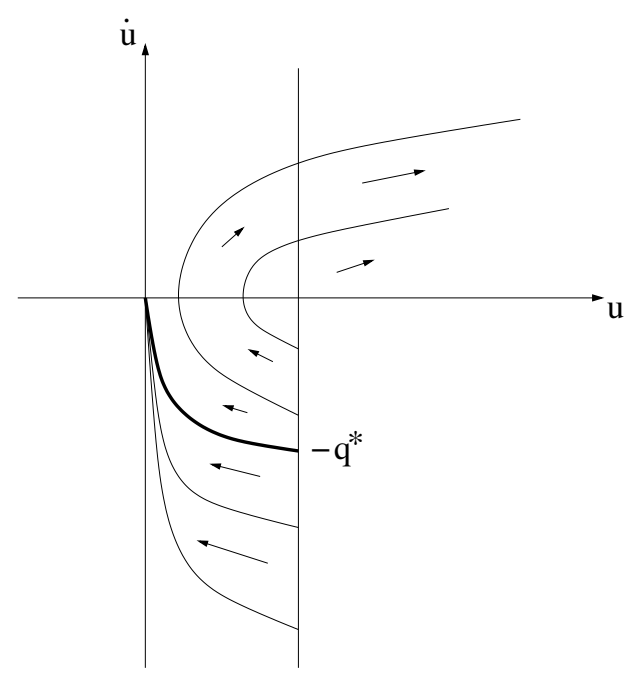

Figure 3: Phase portrait for equation (3.5). The thick line is the solution for initial derivative $q^{*}$ and separates the region with bounded solutions from the region with unbounded solutions.

We can now define $q^{*}$ by

$$
q^{*}:=\sup Q, \quad Q:=\left\{q \geq 0 \mid \text { the solution } \theta=\theta_{q} \text { is unbounded }\right\} .
$$

For $q=0$, the solution $\theta$ is increasing on $\left(0, y_{0}\right)$ and unbounded. In particular we conclude that the set $Q$ is not empty. The claim in the second step below verifies that the set $Q$ is bounded. These two observations show that $q^{*} \in(0, \infty)$ is well-defined. It remains to observe that for two number $q_{2}>q_{1} \geq 0$ holds: If the solution $\theta_{2}$ corresponding to $q_{2}$ is unbounded, then also the solution 
$\theta_{1}$ corresponding to $q_{1}$ is unbounded. This can be concluded from the phase portrait, formally by concluding $\partial_{y} \theta_{2}(y)-\left(\partial_{y} \theta_{1}\right)(Y(y)) \leq 0$, where $Y(y)$ is chosen such that $\theta_{2}(y)=\theta_{1}(Y(y))$.

Second step. Solutions with fast decay. The aim of this step is to verify the following

Claim: For $q^{2} \geq 4 c$ the solution $\theta_{q}$ satisfies, for all $y \in(0, \infty)$,

$$
\begin{aligned}
0<\theta_{q}(y) & \leq e^{-\frac{q}{2} y}, \\
\frac{\partial_{y} \theta_{q}}{\theta_{q}} & <-\frac{q}{2} .
\end{aligned}
$$

Proof of the claim. Assume that (i) is satisfied on $\left(0, y_{0}\right)$. We claim that this implies that also (ii) is satisfied until $y_{0}$. Indeed, the derivative satisfies

$$
\partial_{y}\left(\frac{\partial_{y} \theta_{q}}{\theta_{q}}\right)=\frac{\partial_{y}^{2} \theta_{q}}{\theta_{q}}-\frac{\left(\partial_{y} \theta_{q}\right)^{2}}{\left(\theta_{q}\right)^{2}}=c \theta_{q} \leq c e^{-\frac{q}{2} y} .
$$

Initially, we have $\frac{\partial_{y} \theta_{q}}{\theta_{q}}(0)=-q$. This provides the estimate

$$
\frac{\partial_{y} \theta_{q}}{\theta_{q}}(y) \leq-q+\int_{0}^{y} c e^{-\frac{q}{2} y} d y<-q+\frac{2 c}{q} \leq-\frac{q}{2} .
$$

On the other hand, let (ii) be satisfied until $y_{0}$. Then

$$
\partial_{y} \theta_{q}<-\frac{q}{2} \theta_{q}
$$

implies that (i) is strictly satisfied until $y_{0}$ and is, by continuity, also satisfied on a larger interval. We conclude that (i) and (ii) are satisfied on $\mathbb{R}_{+}$and have thus verified the claim.

The dependence $q^{*}(c)=q^{*}(1) \sqrt{c}$ follows immediately by a scaling argument with the substitution $z=\sqrt{c} y$.

Third step. Limit of the production rate. We keep $c$ fixed and omit the $c$ dependence in the following. For the sequence of solutions $\eta=\bar{\eta}^{k}$ of (3.1)-(3.3) we consider

$$
\theta=\theta_{k}=\frac{e^{\eta / b}}{e^{k / b}}, \quad y_{0}=y_{0}(k)=\delta(k) e^{k /(2 b)},
$$

such that $\theta$ solves (3.5)-(3.7) on $\left(0, y_{0}\right)$. The unknown value $q=q_{k}=-\partial_{y} \theta_{k}(0)$ is determined by the boundary condition $\partial_{y} \theta_{k}\left(y_{0}\right)=0$. We note that $\theta_{k} \geq 0$ holds by definition of $\theta$ and that $\theta_{k}$ is monotonically decreasing on $\left(0, y_{0}\right)$ because of $\partial_{y} \theta_{k}\left(y_{0}\right)=0$. The initial condition satisfies $q_{k} \leq q^{*}$, since a continuation of the solution $\theta_{k}$ beyond $y_{0}$ is necessarily unbounded. The boundedness of the 


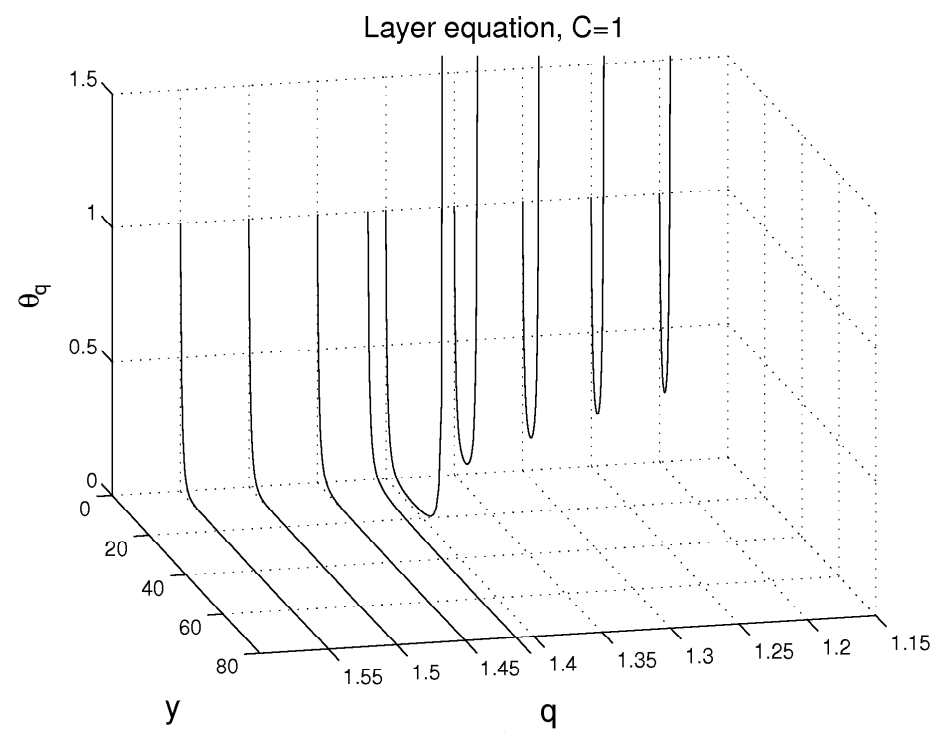

Figure 4: Numerical solutions of system (3.5)-(3.7). The results were obtained by M. Mihailovici using MATLAB.

derivatives $q_{k}$ allows to choose a subsequence $k \rightarrow \infty$ such that $q_{k} \rightarrow q_{\infty}$ for some limiting value $q_{\infty} \leq q^{*}$.

The functions $\theta_{k}$, defined on $\left(0, y_{0}(k)\right)$ with $y_{0}(k) \rightarrow \infty$, are uniformly bounded with uniformly bounded second derivatives. We may therefore assume that our subsequence satisfies $\theta_{k} \rightarrow \theta_{\infty}$ uniformly on compact subsets of $[0, \infty)$. By the boundedness of the second derivatives, also the first derivatives converge uniformly and we find $-q_{\infty} \leftarrow-q_{k}=\partial_{y} \theta_{k}(0) \rightarrow \partial_{y} \theta_{\infty}(0)$. The limiting function $\theta_{\infty}$ inherits the properties of $\theta_{k}$ : it is a solution of (3.5) and it is bounded by 1 . This implies $q_{\infty} \geq q^{*}$ and we have thus verified $q_{k} \rightarrow q_{\infty}=q^{*}$.

The production rate is

$$
\bar{F}_{k}=-\partial_{x} \bar{\eta}^{k}(0)=-\left.e^{k /(2 b)} b \partial_{y}\left[\log \left(\theta_{k} e^{k / b}\right)\right]\right|_{y=0}=-b e^{k /(2 b)} \frac{\partial_{y} \theta_{k}(0)}{\theta_{k}(0)}
$$

hence

$$
\frac{\bar{F}_{k}}{e^{k /(2 b)}}=-b \partial_{y} \theta_{k}(0)=b q_{k} \underset{k \rightarrow \infty}{\longrightarrow} b q^{*}=b q_{1} \sqrt{c}
$$

Recalling $c=\mu_{\eta} \bar{C} / b$, this concludes the proof of (3.8).

Numerically, we determined the value $q_{1}=1.413 \pm 0.002$. We refer to Figure 4 for the shape of solutions for various values of $q$. 


\subsection{Asymptotic behavior in case of large oxygen diffu- sion}

The aim of this subsection is to determine the asymptotic behavior of the total production $F_{k}$ for the coupled system of equations (1.3)-(1.6) in the limit $k \rightarrow$ $\infty$. In the case of a small reaction constant $\mu_{C}=\mu_{C}(k)$ we are able to compare these solutions $\left(C^{k}, \eta^{k}\right)$, in the catalyst layer, with a family of solutions $\left(\bar{C}, \bar{\eta}^{k}\right)$ of (3.1)-(3.3). The subsequent proposition concludes the proof of Theorem 1.

Proposition 2. Let $\mu_{\eta}>0$, let $k \rightarrow \infty$ be a sequence and $\delta=\delta(k), \mu_{C}=\mu_{C}(k)$ be parameters with $\delta(k) \rightarrow \delta_{0} \in[0,1), \delta(k) e^{k /(2 b)} \rightarrow \infty, \mu_{C}(k) e^{k /(2 b)} \rightarrow \bar{\mu}_{C} \in$ $[0, \infty)$, and $\delta(k)^{5} e^{5 k /(2 b)} \mu_{C}(k) \rightarrow 0$. Let $\left(\eta^{k}, C^{k}\right)$ be the corresponding solution of (1.3)-(1.6). Let the real number $\bar{C} \geq 0$ be the unique solution of

$$
\bar{C}+q_{1} \bar{\mu}_{C}\left(1-\delta_{0}\right) \sqrt{b \bar{C} / \mu_{\eta}}=C_{1}
$$

and $\bar{\eta}^{k}$ be the solution of (3.1)-(3.3), according to $\bar{C}$. Then the following convergences hold.

(i) $\left\|C^{k}-\bar{C}\right\|_{L^{\infty}((0, \delta(k)))} \rightarrow 0$ and $\left\|\eta^{k}-\bar{\eta}^{k}\right\|_{L^{\infty}((0, \delta(k)))} \rightarrow 0$ for $k \rightarrow \infty$,

(ii) $\frac{F_{k}}{e^{k /(2 b)}} \rightarrow q_{1} \sqrt{b \mu_{\eta} \bar{C}}$ for $k \rightarrow \infty$.

Proof. We first note that from the assumptions we also have the decays $\delta^{2}(k) e^{k / b} \mu_{C}(k) \rightarrow 0, \delta^{3}(k) e^{3 k /(2 b)} \mu_{C}(k) \rightarrow 0$, and $\delta^{4} e^{2 k / b} \mu_{C}(k)$ as $k \rightarrow \infty$.

First step. Convergence of $C^{k}$. From the maximum principle we know the bounds $0 \leq C^{k} \leq C_{1}$ and $\eta^{k}, \bar{\eta}^{k} \leq k$. In particular, we have $C^{k}(0) \in\left[0, C_{1}\right]$ and we find a subsequence and $\bar{C} \in\left[0, C_{1}\right]$ such that $C^{k}(0) \rightarrow \bar{C}$ for $k \rightarrow \infty$. We will later derive the characterizing relation (3.10) for this limit $\bar{C}$.

Integrating equation (1.5) we obtain for any $x \in \Omega_{0}$

$$
\partial_{x} C^{k}(x)=\int_{0}^{x} \mu_{C}(k) C^{k} e^{\eta^{k} / b} \mathbf{1}_{\Omega_{\delta}} \leq \delta \mu_{C}(k) C_{1} e^{k / b}
$$

and therefore, for all $x \in[0, \delta]$,

$$
C^{k}(x)-C^{k}(0)=\int_{0}^{x} \partial_{x} C^{k} \leq \delta^{2} \mu_{C}(k) C_{1} e^{k / b}
$$

By monotonicity of $C^{k}$ we find, in particular, the $C^{k}$-convergence of (i).

Second step. Convergence of $\eta^{k}$. We subtract the equations (1.3) and (3.1) and test with the difference $\left(\eta^{k}-\bar{\eta}^{k}\right)$. We obtain

$$
\int_{0}^{\delta}\left|\partial_{x}\left(\eta^{k}-\bar{\eta}^{k}\right)\right|^{2}=\int_{0}^{\delta}\left(\bar{C}-C^{k}\right) e^{\eta^{k} / b}\left(\eta^{k}-\bar{\eta}^{k}\right)-\bar{C} \int_{0}^{\delta}\left(e^{\eta^{k} / b}-e^{\bar{\eta}^{k} / b}\right)\left(\eta^{k}-\bar{\eta}^{k}\right) .
$$


Due to the monotonicity of the exponential function the second integral on the right-hand side is nonnegative such that Hölder's and Poincaré's inequality allow to estimate

$$
\begin{array}{r}
\int_{0}^{\delta}\left|\partial_{x}\left(\eta^{k}-\bar{\eta}^{k}\right)\right|^{2} \leq \int_{0}^{\delta}\left(\bar{C}-C^{k}\right) e^{\eta^{k} / b}\left(\eta^{k}-\bar{\eta}^{k}\right) \\
\leq \delta^{3 / 2} e^{k / b}\left\|\bar{C}-C^{k}\right\|_{\infty}\left\|\partial_{x}\left(\eta^{k}-\bar{\eta}^{k}\right)\right\|_{L^{2}\left(\Omega_{\delta}\right)}
\end{array}
$$

The last estimate implies, with the fundamental theorem of calculus,

$$
\left\|\eta^{k}-\bar{\eta}^{k}\right\|_{\infty} \leq \delta^{1 / 2}\left\|\partial_{x}\left(\eta^{k}-\bar{\eta}^{k}\right)\right\|_{L^{2}\left(\Omega_{\delta}\right)} \leq \delta^{2} e^{k / b}\left\|\bar{C}-C^{k}\right\|_{\infty} \leq \delta^{4} \mu_{C}(k) C_{1} e^{2 k / b} .
$$

We find, in particular, the $\eta^{k}$-convergence of (i).

Third step. Convergence of $F_{k}$. Comparing the total production $F_{k}$ corresponding to $\eta^{k}$ with the production $\bar{F}_{k}$ corresponding to $\bar{\eta}^{k}$ we obtain

$$
\frac{1}{\mu_{\eta}}\left|F_{k}-\bar{F}_{k}\right|=\left|\int_{0}^{\delta}\left(C^{k} e^{\eta^{k} / b}-\bar{C} e^{\bar{\eta}^{k} / b}\right)\right| \leq \delta e^{k / b}\left\|C^{k}-\bar{C}\right\|_{\infty}+\delta C_{1}\left\|e^{\eta^{k} / b}-e^{\bar{\eta}^{k} / b}\right\|_{\infty} .
$$

Since $\left|e^{\eta^{k}(x) / b}-e^{\bar{\eta}^{k}(x) / b}\right| \leq \frac{1}{b} e^{k / b}\left|\eta^{k}(x)-\bar{\eta}^{k}(x)\right|$ for all $x \in \Omega_{\delta}$ we conclude

$$
\frac{1}{\mu_{\eta}}\left|F_{k}-\bar{F}_{k}\right| \leq \delta e^{k / b}\left\|C^{k}-\bar{C}\right\|_{\infty}+\delta \frac{C_{1}}{b} e^{k / b}\left\|\eta^{k}-\bar{\eta}^{k}\right\|_{\infty}
$$

and from the results of the first and second step we have

$$
\frac{1}{\mu_{\eta}}\left|F_{k}-\bar{F}_{k}\right| \leq \delta^{3} C_{1} \mu_{C}(k) e^{2 k / b}+\delta^{5} \frac{C_{1}^{2}}{b} \mu_{C}(k) e^{3 k / b} .
$$

Finally, with the assumption on the decay of $\delta(k)^{5} \mu_{C}(k)$, we obtain

$$
\left|\frac{F_{k}}{e^{k /(2 b)}}-q_{1} \sqrt{b \mu_{\eta} \bar{C}}\right| \leq\left|\frac{F_{k}}{e^{k /(2 b)}}-\frac{\bar{F}_{k}}{e^{k /(2 b)}}\right|+\left|\frac{\bar{F}_{k}}{e^{k /(2 b)}}-q_{1} \sqrt{b \mu_{\eta} \bar{C}}\right|
$$

where the right-hand side tends to 0 as $k \rightarrow \infty$ due to the estimate (3.11) and Proposition 1. This shows (ii).

Fourth step. Characterization of $\bar{C}$. Since $\partial_{x}^{2} C^{k}=0$ on $(\delta, 1)$, the first derivative of $C^{k}$ is constant on $(\delta, 1)$ and we obtain

$$
\partial_{x} C^{k}(\delta)=\frac{C_{1}-C^{k}(\delta)}{1-\delta}
$$

Due to the definition of the total production we can calculate

$$
\begin{aligned}
\bar{\mu}_{C} q_{1} \sqrt{b \mu_{\eta} \bar{C}} \leftarrow \mu_{C}(k) F_{k} & =\mu_{\eta} \int_{0}^{\delta} \partial_{x}^{2} C^{k}=\mu_{\eta} \partial_{x} C^{k}(\delta) \\
& =\mu_{\eta} \frac{C_{1}-C^{k}(\delta)}{1-\delta} \rightarrow \mu_{\eta} \frac{C_{1}-\bar{C}}{1-\delta_{0}}
\end{aligned}
$$

by the first step. 
Acknowledgment. The authors gratefully acknowledge the support by the Swiss grant SNF 03JANCHD and the German grant BMBF 03SF0310B.

\section{References}

[1] A. Bourgeat, O. Gipouloux, and E. Marušić-Paloka. Modelling of an underground waste disposal site by upscaling. Math. Methods Appl. Sci., 27(4):381-403, 2004.

[2] Y. Bultel, P. Ozil, and R. Durand. Concentration and potential distributions in the active layer of proton exchange membrane fuel cell electrodes. J. Appl. Electrochem., 30(12):1369-1376, 2000.

[3] G. Caginalp and W. Xie. An analysis of phase-field alloys and transition layers. Arch. Rational Mech. Anal., 142(4):293-329, 1998.

[4] A. Capella, Chr. Melcher, and F. Otto. Wave-type dynamics in ferromagnetic thin film and the motion of néel walls. Nonlinearity, 20:2519-2537, 2007.

[5] S.H. Chan and W.A. Tun. Catalyst layer models for Proton Exchange Membrane Fuel Cells. Chem. Eng. Technol., 24(1):51-57, 2001.

[6] P. Colli and J. Rodrigues. Diffusion through thin layers with high specific heat. Asymptotic Anal., 3(3):249-263, 1990.

[7] M. Eikerling and A.A. Kornyshev. Modelling the performance of the cathode catalyst layer of polymer electrolyte fuel cells. J. Electroanal. Chem., 453:89-106, 1998.

[8] H. Garcke, B. Nestler, and B. Stinner. A diffuse interface model for alloys with multiple components and phases. SIAM J. Appl. Math., 64(3):775-799 (electronic), 2004.

[9] F. Jaouen, G. Lindbergh, and G. Sundholm. Investigation of masstransport limitations in the solid polymer fuel cell cathode. J. Electrochem. Soc., 149(4):A437-A447, 2002.

[10] A.A. Kulikovsky. Quasi three-dimensional modelling of the PEM fuel cell: Comparison of the catalyst layers performance. Fuel Cells, 1(2):162-169, 2001.

[11] M. Mihailovici and B. Schweizer. Effective model for the cathode catalyst layer in fuel cells. Asymptot. Anal., 57(1-2):105-123, 2008. 
[12] St. Müller and M. R. Pakzad. Convergence of equilibria of thin elastic plates - the von Kármán case. Comm. Partial Differential Equations, 33(46):1018-1032, 2008.

[13] M. Neuss-Radu and W. Jäger. Effective transmission conditions for reaction-diffusion processes in domains separated by an interface. SIAM J. Math. Anal., 39(3):687-720 (electronic), 2007.

[14] M.L. Perry, J. Newman, and E.J. Cairns. Mass transport in gas-diffusion electrodes: A diagnostic tool for fuel-cell cathodes. J. Electrochem. Soc., 145(1):5-15, 1998.

[15] J.X. Wang, F.A. Uribe, T.E. Springer, J. Zhang, and R.R. Adzic. Intrinsic kinetic equation for oxygen reduction reaction in acidic media: the double tafel slope and fuel cell applications. Faraday Discuss., 2009.

[16] K.Z. Yao, K. Karan, K.B. McAuley, P. Oosthuizen, B. Peppley, and T. Xie. A review of mathematical models for hydrogen and direct methanol polymer electrolyte membrane fuel cells. Fuel Cells, 4(1-2):3-29, 2004. 


\section{Preprints ab 2008}

2008-01 Henryk Zähle

Weak approximation of SDEs by discrete-time processes

2008-02 Benjamin Fine, Gerhard Rosenberger

An Epic Drama: The Development of the Prime Number Theorem

2008-03 Benjamin Fine, Miriam Hahn, Alexander Hulpke, Volkmar große Rebel, Gerhard Rosenberger, Martin Scheer

All Finite Generalized Tetrahedron Groups

2008-04

2008-05

2008-06

Ben Schweizer

Homogenization of the Prager model in one-dimensional plasticity

Benjamin Fine, Alexei Myasnikov, Gerhard Rosenberger

Generic Subgroups of Group Amalgams

Flavius Guiaş

Generalized Becker-Döring Equations Modeling the Time Evolution of a Process of Preferential Attachment with Fitness

2008-07 Karl Friedrich Siburg, Pavel A. Stoimenov

A scalar product for copulas

2008-08 Karl Friedrich Siburg, Pavel A. Stoimenov

A measure of mutual complete dependence

2008-09 Karl Friedrich Siburg, Pavel A. Stoimenov

Gluing copulas

2008-10 Peter Becker-Kern, Wilfried Hazod

Mehler hemigroups and embedding of discrete skew convolution semigroups on simply connected nilpotent Lie groups

2008-11 Karl Friedrich Siburg

Geometric proofs of the two-dimensional Borsuk-Ulam theorem

2008-12 Michael Lenzinger and Ben Schweizer

Two-phase flow equations with outflow boundary conditions in the hydrophobic-hydrophilic case

2008-13 Wilfried Hazod

Probability on Matrix-Cone Hypergroups: Limit Theorems and

Structural Properties

2008-14 Wilfried Hazod

Mixing of generating functionals and applications to (semi-)stability

of probabilities on groups 
Wilfried Hazod

Multiple selfdecomposable laws on vector spaces and on groups:

The existence of background driving processes

2008-16 Guy Bouchitté and Ben Schweizer

Homogenization of Maxwell's equations with split rings

2008-17 Ansgar Steland and Henryk Zähle

Sampling inspection by variables: nonparametric setting

2008-18 Michael Voit

Limit theorems for radial random walks on homogeneous spaces with growing dimensions

2008-19 Michael Voit

Central Limit Theorems for Radial Random Walks on

$p \times q$ Matrices for $p \rightarrow \infty$

2008-20 Margit Rösler and Michael Voit

Limit theorems for radial random walks on $p \times q$-matrices as $p$ tends to infinity

2008-21 Michael Voit

Bessel convolutions on matrix cones: Algebraic properties and random walks

2008-22 Michael Lenzinger and Ben Schweizer

Effective reaction rates of a thin catalyst layer 